\title{
On the gamma-ray burst - gravitational wave association in GW150914
}

\author{
Agnieszka Janiuk $^{1}$, Szymon Charzynski ${ }^{2}$ and Michal Bejger ${ }^{3}$ \\ ${ }^{1}$ Center for Theoretical Physics, Polish Academy of Sciences, \\ Al. Lotnikow 32/46, 02-668 Warsaw, Poland \\ email: agnes@cft.edu.pl \\ ${ }^{2}$ Chair of Mathematical Methods in Physics, University of Warsaw \\ ul. Pasteura 5, 02-093 Warszawa, Poland \\ email: szycha@fuw.edu.pl \\ ${ }^{3}$ Copernicus Astronomical Center, Polish Academy of Sciences, \\ Bartycka 18, 00-716 Warsaw, Poland \\ email: bajger@camk.edu.pl
}

\begin{abstract}
Hyperaccreting disks around black holes are the engines that drive outflows and jets in gamma ray bursts (GRBs). The torus formed after the core collapse or a compact binary merger is composed of free nucleons, Helium, electron-positron pairs, and is cooled by neutrinos rather than photon emission. Hyperaccretion powers the ultra-relativistic jets, where the GRB prompt emission originates. The neutrons produced in the disk and also in the outflowing material are necessary for the production of heavier nuclei. We discuss here the observable consequences of nucleosynthesis and we also apply the scenario of hyperaccretion to the gravitational wave source, GW150914. Temporal coincidence reported by the Fermi satellite suggested that the black hole merger might be accompanied with a GRB. We propose that a collapsing massive star and a black hole in a close binary could lead to such event. Gravitational wave emission due to the merger of collapsed core and the companion black hole might then coincide with a weak GRB.
\end{abstract}

Keywords. Black hole physics, accretion disks, gamma ray bursts, gravitational waves, neutrinos

\section{Introduction}

Gamma Ray Bursts (GRBs), are extremely energetic, transient events, visible from the most distant parts of the Universe. When a newly born black hole forms, either via the core collapse supernova, or after the merger of two compact stars, a large amount of matter is accreted onto it within the timescale between tens of milliseconds, to thousands of seconds, with highly hyper-Eddington rates. In such conditions, the nuclear reactions inside the plasma lead to creation of neutrinos, mainly via the electron-positron capture on nucleons. The annihilation of neutrino-antineutrino pairs is a source of power to the relativistic jets launched along the axis of the disk-black hole system. This can be comparable to the power extracted at the cost of the black hole spin, with the disk mediated by the magnetic fields. Both neutrinos and magnetic fields in the accreting matter can drive the wind outflow from the disk surface, and act as a collimation mechanism for the relativistic jets (e.g., Popham et al. 1999, Di Matteo et al. 2002, Janiuk et al. 2007), Janiuk \& Yuan 2010). More recently, accretion flows in GRBs were described by fully relativistic, magneto-hydrodynamic (MHD) computations (e.g., Barkov \& Komissarov 2010, Barkov \& Baushev 2011, Janiuk et al. 2013a). 
In 2013, before the gravitational waves were detected, we have speculated, that in a certain type of progenitors, such as the high -mass X-ray binaries, the core collapse of a massive star might coincide with the companion black hole entering the star's envelope (Janiuk et al. 2013b). In this scenario, the collapsing core is spun up by the transfer of angular momentum from the companion, and the merger of a spinning black hole just after the collapse, with this companion black hole, produces the gravitational wave signal. The event can be accompanied by the gamma ray burst, if one, or two jets are launched at the cost of accretion onto a rotating black hole. The GRB may be observed prior, and/or after the black hole merger, depending on the black hole spin, hence the jet orientation with respect to the line of sight.

\section{Black hole meger and the hyper-accretion flow for GW 150914}

The first discovered source of gravitational waves, GW150914 event, was interpreted to be a merger of two black holes (BHs) of the masses of $36_{-4}^{+5} M_{\odot}$ and $29_{-4}^{+4}$. The final BH mass was $62_{-4}^{+4} M_{\odot}$ and its spin was $0.67_{-0.07}^{+0.05}$ (Abbott et al. 2016). Probabilities that the angles between spins and the normal to the orbital plane are between $45^{\circ}$ and $135^{\circ}$ are about 0.8 for each component $\mathrm{BH}$, while the spin magnitudes are smaller than 0.7 and 0.8 at $90 \%$ probability. Assumption of a strict co-alignment of spins with the orbital angular momentum results in an upper limit of 0.2 and 0.3 for the spins. The Fermi satellite detected a weak gamma ray signal coincident with the GW event (Connaughton et al. 2016).

Binary black hole merger simulations and constraints for the engine. We made several runs for BH mass and range of spins, constrained from the Advanced LIGO data. These simulations were performed in vacuum approximation, and the numerical package we used is Einstein Toolkit† It is a family of codes for use in relativistic astrophysics, based on finite difference computation on a gridded mesh (Loeffler et al. 2012), supported by a distributed model, combining core support of software, tools, and documentation. We use the source code which we configured, compiled and run in the computer cluster in the Warsaw University (ICM). The code is developed on the basis of the theoretical achievements of the last decade, which made possible to establish the field of numerical relativity (see also, e.g., Pretorius 2005, Campanelli et al. 2006).

In Figure 1, on the left, we plot the example result from the run with $\mathrm{BH}$ mass ratio of 0.82 , and aligned spins of 0.2 and 0.3 . The waveform is plotted using the Weyl scalar $\psi_{4}$. The final $\mathrm{BH}$ spin obtained in this simulation was 0.68 . On the right, we show the $3-\mathrm{D}$ visualisation of the torus accreting onto a single black hole, which presumably formed after the merger, and was composed from the remnants of the star's envelope.

GRB central engine. To model the accretion and the GRB engine, we use the code

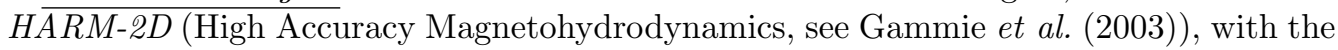
numerical EOS taken from Janiuk et al. (2007), and neutrino cooling implemented into MHD evolution. The black hole parameters were constrained by the post-merger conditions, and the torus parameters matched by the weak GRB properties. From our simulation, we estimate the neutrino and Blandford-Znajek luminosities available to power the GRB jet in this source (see Janiuk et al. 2017, and Janiuk 2016 for details).

The HARM code works in the stationary metric around black hole and integrates the total energy equation and updates the set of 'conserved' variables, i.e. comoving density, energy-momentum, and magnetic field, assuming a force-free approximation. Here, we first incorporate this detailed microphysics into the heart of the general relativistic

$\dagger$ http://www.einsteintoolkit.org 


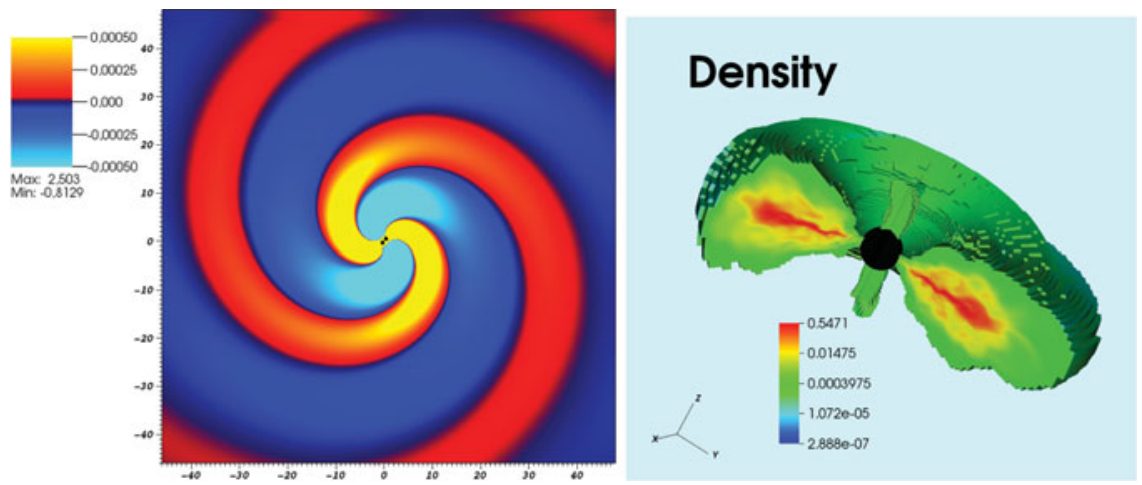

Figure 1. The spacetime curvature, depicted in the orbital plane, just before the black hole merger (left). The plotted quantity is the Weyl scalar $\psi_{4}$, which shows the outgoing gravitational radiation for the asymptotically flat spacetime. Distribution of density in the torus accreting onto a spinning black hole (right). The $\mathrm{BH}$ in this scenario is a product of the binary merger.

MHD scheme. We allow for the partial degeneracy of relativistic nucleons, electrons and positrons in the accreting plasma, and we compute the neutrino cooling rate due to the weak interaction processes, electron-positron pair annihilation, as well as bremsstrahlung and plasmon decay.

We found that for the $\mathrm{BH}$ mass of $M=62 M_{\odot}$, the spin $a=0.7$, and disk mass $M_{\mathrm{d}}=15 M_{\odot}$, and also assuming the low efficiencies of conversion between neutrino annihilation, and conversion between the jet kinetic and radiative power, this scenario is able to meet quantitatively the limits put by the Fermi data.

Neutrino emission from GRB engine and efficiency of jet power supply. The maximum of the neutrino luminosity obtained in our simulations is reached about 0.4 seconds after an equilibrium torus, prescribed by our initial conditions, had formed. This may tentatively give the lower limit for the timescale when the jet appears after the BH merger. The Blandford-Znajek luminosity is of the order of $10^{50}-10^{51} \mathrm{ergs} / \mathrm{s}$, and only occasionally non-zero for low spins (the jet is powered by magnetic field only episodically). We find therefore, that the GRB luminosity inferred from our simulations can be reconciled with the observational upper limits, for moderate spins of the final $\mathrm{BH}(a=0.6-0.8)$.

\section{Heavy elements produced in the GRB engine}

In the outer parts of the accretion flow in the GRB central engine, the heavy elements can be formed, and then the isotopes are prone to the radioactive decay. We compute the abundance pattern of these elements with the thermonuclear reaction network code, based on the profiles of density, temperature, and electron fraction in the accretion torus, obtained in our HARM simulations. We determined the abundances of heavy elements (above Helium, up to the mass number of $\sim 130$ ), which are synthesized. The decay of some of the unstable isotopes may in the future bring observable effects on the measured hard X-ray spectra of GRBs (Janiuk 2014) as well as the additional peaks in the blue and infrared bands a few days after the prompt GRB emission. The observational signatures of radioactive decay of some of the unstable isotopes can be, for instance, the emission lines seen in the X-rays (in principle, in the $N u S T A R$ range), or in the faint emission in lower energy band continuum (i.e. the 'macronova' scenario). In addition, the gamma ray bursts contribute in this way to the galactic chemical evolution.

The physical conditions in the engine depend on the global parameters of the flow, such as the amount of matter and accretion rate, black hole mass, its spin, and the 
magnetic field strength. These conditions in turn will affect the total abundances of heavier elements. Therefore, a detection of such gamma ray burst signatures, apart from its prompt fluence, could give an independent constraint on the black hole parameters. These, in turn, can now be also estimated, if the black hole was born via a merger detectable by the gravitational wave interferometer.

Constraints from and for the gravitational wave astronomy. So far, for GW150914, the electromagnetic counterpart was tentatively reported only by the Fermi satellite, which detected a weak gamma ray signal. No infrared emission was reported, and also the signal was not confirmed by other gamma-ray missions for the case of this burst (Greiner et al. 2016). Nevertheless, the detection of the electromagnetic counterpart of a gravitational wave source in the future events is appealing.

In the case of the relatively small rate of the black hole rotation, such as estimated for GW150914, the hyperaccretion results in a rather small electromagnetic flux dragged through the black hole horizon and hence small power of the Blandford-Znajek process. The gravitational waves discovery may reveal a new population of weak gamma ray bursts originating from the low Lorentz factor jets. In this case, the prompt electromagnetic emission may be difficult to discover, but the observation of a radioactive decay of elements produced in the engine may be confronted with our simulation predictions. The black hole parameters, its spin and mass, determine the shape of the gravitational wave signal.

\section{Acknowledgements}

This work was supported in part by the grants no. DEC-2012/05/E/ST9/03914 and UMO-2014/14/M/ST9/00707 from the Polish National Science Center. We also acknowledge support from the Interdisciplinary Center for Mathematical Modeling of the Warsaw University, through the computational grant G53-5.

\section{References}

Abbott, B. P., et al., 2016, Phys. Rev. Lett., 116, 061102

Barkov, M. V. \& Baushev, A. N., 2011, New Astr., 16, 46

Barkov, M. V. \& Komissarov, S. S., 2010, MNRAS, 401, 1644

Blandford, R. D., \& Znajek, R. L., 1977, MNRAS, 179, 433

Campanelli, M., Lousto, C. O., Marronetti, P., \& Zlochower, Y., 2006, Phys. Rev. Lett., 96, 11

Connaughton, V., et al., 2016, ApJ Lett., 826, 6

Di Matteo, T., Perna, R., \& Narayan, R., 2002, ApJ, 579, 706

Fishbone, L. G., \& Moncrief, V., 1976, ApJ, 207, 962

Gammie, C. F., McKinney, J. C., \& Tth, G., 2003, ApJ, 589, 444

Greiner, J, Burgess, J. M., Savchenko, V., \& Yu, H.-F., 2016, ApJ Lett., 827, 38

Janiuk, A., Bejger, M., Charzynski, S., \& Sukova, P., 2017, New Astr, 51, 7

Janiuk, A., 2014, A\&A, 568, 105

Janiuk, A., Mioduszewski, P., \& Moscibrodzka, M., 2013, ApJ, 776, 105

Janiuk, A., Charzynski, S., \& Bejger, M., 2013b, A\&A, 560, 25

Janiuk, A., \& Yuan, Y. F., 2010, A\&A A, 509, 55

Janiuk, A., Yuan, Y. F., Perna, R., \& Di Matteo, T., 2007, ApJ, 664, 1011

Janiuk, A., 2016, submitted, arXiv:1609.09361

Löffler, F., et al. 2012, Class. Q. Grav., 29, 11

Li, L. X., \& Paczynski, B. P., 1998, ApJ, 507, 59

Mc Kinney, J. C., \& Gammie, C. F. , 2004, ApJ, 611, 977

Noble, S. C., Gammie, C. F., McKinney, J. C., \& Del Zanna, L., 2006, ApJ, 641, 626

Pretorius F., 2005, Class. Q. Grav., 22, 425

Popham, R., Woosley, S. E. \& Fryer, C. 1999, ApJ, 518, 356 